\title{
SPLITTINGS AND DISJUNCTIONS IN REVERSE MATHEMATICS
}

\author{
SAM SANDERS
}

\begin{abstract}
Reverse Mathematics (RM hereafter) is a program in the foundations of mathematics founded by Friedman and developed extensively by Simpson and others. The aim of RM is to find the minimal axioms needed to prove a theorem of ordinary, i.e. non-set-theoretic, mathematics. As suggested by the title, this paper deals with two (relatively rare) RM-phenomena, namely splittings and disjunctions. As to splittings, there are some examples in RM of theorems $A, B, C$ such that $A \leftrightarrow(B \wedge C)$, i.e. $A$ can be split into two independent (fairly natural) parts $B$ and $C$. As to disjunctions, there are (very few) examples in RM of theorems $D, E, F$ such that $D \leftrightarrow(E \vee F)$, i.e. $D$ can be written as the disjunction of two independent (fairly natural) parts $E$ and $F$. By contrast, we show in this paper that there is a plethora of (natural) splittings and disjunctions in Kohlenbach's higher-order RM.
\end{abstract}

\section{INTRODUCTION}

Reverse Mathematics (RM hereafter) is a program in the foundations of mathematics initiated around 1975 by Friedman $([19,20])$ and developed extensively by Simpson ([58) and others. We refer to [60] for a basic introduction to RM and to [57,58, for an (updated) overview of RM. We will assume basic familiarity with RM, the associated 'Big Five' systems and the 'RM zoo' ([18). We do introduce Kohlenbach's higher-order RM in some detail Section 2.1.

As discussed in e.g. [27, §6.4], there are (some) theorems $A, B, C$ in the RM zoo such that $A \leftrightarrow(B \wedge C)$, i.e. $A$ can be split into two independent (fairly natural) parts $B$ and $C$ (over $\mathrm{RCA}_{0}$ ). As to the possibility of $A \leftrightarrow(B \vee C)$, there is [21, Theorem 4.5] which states that a certain theorem about dynamical systems is equivalent to the disjunction of weak König's lemma and induction for $\Sigma_{2}^{0}$-formulas; neither disjunct of course implies the other $\left(\right.$ over $\left.R C A_{0}\right)$. Similar results are in 8 for model theory, but these are more logical in nature.

It is fair to say that there are only few natural examples of splittings and disjunctions in RM, though such claims are invariably subjective in nature. Nonetheless, the aim of this paper is to establish a plethora of splittings and disjunctions in higher-order RM. In particular, we obtain splittings and disjunctions involving (higher-order) $\mathrm{WWKL}_{0}$, the Big Five, and $\mathrm{Z}_{2}$, among others. We similarly treat the covering theorems Cousin's lemma and Lindelöf's lemma studied in [50]. Our main results are in Section 3, while a summary may be found in Section 5 , our base theories are generally conservative over $\mathrm{WKL}_{0}$ (or are strictly weaker).

It goes without saying that our results highlight a major difference between second- and higher-order arithmetic, and the associated development of RM. We provide some musings on this and related foundational matters in Section 4.

School of Mathematics, University of Leeds \& Dept. of Mathematics, TU Darmstadt

E-mail address: sasander@me.com. 


\section{Preliminaries}

2.1. Higher-order Reverse Mathematics. We sketch Kohlenbach's higher-order Reverse Mathematics as introduced in [34. In contrast to 'classical' RM, higherorder RM makes use of the much richer language of higher-order arithmetic.

As suggested by its name, higher-order arithmetic extends second-order arithmetic. Indeed, while the latter is restricted to numbers and sets of numbers, higherorder arithmetic also has sets of sets of numbers, sets of sets of sets of numbers, et cetera. To formalise this idea, we introduce the collection of all finite types $\mathbf{T}$, defined by the two clauses:

(i) $0 \in \mathbf{T}$ and (ii) If $\sigma, \tau \in \mathbf{T}$ then $(\sigma \rightarrow \tau) \in \mathbf{T}$,

where 0 is the type of natural numbers, and $\sigma \rightarrow \tau$ is the type of mappings from objects of type $\sigma$ to objects of type $\tau$. In this way, $1 \equiv 0 \rightarrow 0$ is the type of functions from numbers to numbers, and where $n+1 \equiv n \rightarrow 0$. Viewing sets as given by characteristic functions, we note that $Z_{2}$ only includes objects of type 0 and 1 ; we denote the associated language by $\mathrm{L}_{2}$.

The language $\mathbf{L}_{\omega}$ includes variables $x^{\rho}, y^{\rho}, z^{\rho}, \ldots$ of any finite type $\rho \in \mathbf{T}$. Types may be omitted when they can be inferred from context. The constants of $\mathrm{L}_{\omega}$ includes the type 0 objects 0,1 and $<_{0},+_{0}, \times_{0},=_{0}$ which are intended to have their usual meaning as operations on $\mathbb{N}$. Equality at higher types is defined in terms of ' $={ }_{0}$ ' as follows: for any objects $x^{\tau}, y^{\tau}$, we have

$$
\left[x=_{\tau} y\right] \equiv\left(\forall z_{1}^{\tau_{1}} \ldots z_{k}^{\tau_{k}}\right)\left[x z_{1} \ldots z_{k}=_{0} y z_{1} \ldots z_{k}\right]
$$

if the type $\tau$ is composed as $\tau \equiv\left(\tau_{1} \rightarrow \ldots \rightarrow \tau_{k} \rightarrow 0\right)$. Furthermore, $\mathrm{L}_{\omega}$ also includes the recursor constant $\mathbf{R}_{\sigma}$ for any $\sigma \in \mathbf{T}$, which allows for iteration on type $\sigma$-objects as in the special case (2.2). Formulas and terms are defined as usual.

Definition 2.1. The base theory $\mathrm{RCA}_{0}^{\omega}$ consists of the following axioms:

(a) Basic axioms expressing that $0,1,<_{0},+_{0}, \times_{0}$ form an ordered semi-ring with equality $=_{0}$.

(b) Basic axioms defining the well-known $\Pi$ and $\Sigma$ combinators (aka $K$ and $S$ in [1]), which allow for the definition of $\lambda$-abstraction.

(c) The defining axiom of the recursor constant $\mathbf{R}_{0}$ : For $m^{0}$ and $f^{1}$ :

$$
\mathbf{R}_{0}(f, m, 0):=m \text { and } \mathbf{R}_{0}(f, m, n+1):=f\left(\mathbf{R}_{0}(f, m, n)\right) .
$$

(d) The axiom of extensionality: for all $\rho, \tau \in \mathbf{T}$, we have:

$$
\left(\forall x^{\rho}, y^{\rho}, \varphi^{\rho \rightarrow \tau}\right)\left[x={ }_{\rho} y \rightarrow \varphi(x)={ }_{\tau} \varphi(y)\right] .
$$

(e) The induction axiom for quantifier-fre ${ }^{1}$ formulas of $\mathbf{L}_{\omega}$.

(f) QF-AC ${ }^{1,0}$ : The quantifier-free axiom of choice as in Definition 2.2,

Definition 2.2. The axiom QF-AC consists of the following for all $\sigma, \tau \in \mathbf{T}$ :

$$
\left(\forall x^{\sigma}\right)\left(\exists y^{\tau}\right) A(x, y) \rightarrow\left(\exists Y^{\sigma \rightarrow \tau}\right)\left(\forall x^{\sigma}\right) A(x, Y(x)),
$$

for any quantifier-free formula $A$ in the language of $\mathrm{L}_{\omega}$.

\footnotetext{
${ }^{1}$ To be absolutely clear, variables (of any finite type) are allowed in quantifier-free formulas of the language $\mathrm{L}_{\omega}$ : only quantifiers are banned.
} 
As discussed in [34, §2], $\mathrm{RCA}_{0}^{\omega}$ and $\mathrm{RCA}_{0}$ prove the same sentences 'up to language' as the latter is set-based and the former function-based. Recursion as in (2.2) is called primitive recursion; the class of functionals obtained from $\mathbf{R}_{\rho}$ for all $\rho \in \mathbf{T}$ is called Gödel's system $T$ of all (higher-order) primitive recursive functionals.

We use the usual notations for natural, rational, and real numbers, and the associated functions, as introduced in [34, p. 288-289].

Definition 2.3 (Real numbers and related notions in $\mathrm{RCA}_{0}^{\omega}$ ).

(a) Natural numbers correspond to type zero objects, and we use ' $n^{0}$ ' and ' $n \in \mathbb{N}$ ' interchangeably. Rational numbers are defined as signed quotients of natural numbers, and ' $q \in \mathbb{Q}$ ' and ' $<_{\mathbb{Q}}$ ' have their usual meaning.

(b) Real numbers are represented by fast-converging Cauchy sequences $q_{(\cdot)}$ : $\mathbb{N} \rightarrow \mathbb{Q}$, i.e. such that $\left.\left(\forall n^{0}, i^{0}\right)\left(\mid q_{n}-q_{n+i}\right) \mid<\mathbb{Q} \frac{1}{2^{n}}\right)$. We use the 'hat function' from [34, p. 289] to guarantee that any $f^{1}$ defines a real number.

(c) We write ' $x \in \mathbb{R}$ ' to express that $x^{1}:=\left(q_{(\cdot)}^{1}\right)$ represents a real as in the previous item and write $[x](k):=q_{k}$ for the $k$-th approximation of $x$.

(d) Two reals $x, y$ represented by $q_{(\cdot)}$ and $r_{(\cdot)}$ are equal, denoted $x==_{\mathbb{R}} y$, if $\left(\forall n^{0}\right)\left(\left|q_{n}-r_{n}\right| \leq \frac{1}{2^{n-1}}\right)$. Inequality ' $<\mathbb{R}^{\prime}$ ' is defined similarly. We sometimes omit the subscript ' $\mathbb{R}$ ' if it is clear from context.

(e) Functions $F: \mathbb{R} \rightarrow \mathbb{R}$ are represented by $\Phi^{1 \rightarrow 1}$ mapping equal reals to equal reals, i.e. $(\forall x, y \in \mathbb{R})\left(x=_{\mathbb{R}} y \rightarrow \Phi(x)=_{\mathbb{R}} \Phi(y)\right)$.

(f) The relation ' $x \leq_{\tau} y$ ' is defined as in (2.1) but with ' $\leq_{0}$ ' instead of ' $={ }_{0}$ '. Binary sequences are denoted ' $f{ }^{1}, g^{1} \leq_{1} 1$ ', but also ' $f, g \in C$ ' or ' $f, g \in 2^{\mathbb{N}}$ '.

We now discuss the issue of representations of real numbers.

Remark 2.4. Introductory analysis courses often provide an explicit construction of $\mathbb{R}$ (perhaps in an appendix), while in practice one generally makes use of the axiomatic properties of $\mathbb{R}$, and not the explicit construction. Now, there are a number of different ${ }^{2}$ such constructions: Tao uses Cauchy sequences in his text 64 and discusses decimal expansions in the Appendix [64, §B]. Hewitt-Stromberg also use Cauchy sequences in [26, §5] and discuss Dedekind cuts in the exercises ([26. p. 46]). Rudin uses Dedekind cuts in [55] and mentions that Cauchy sequences yield the same result. Clearly, Definition 2.3 is based on Cauchy sequences, but Hirst has shown that over RCA $\mathrm{A}_{0}$, individual real numbers can be converted between various representations ([28]). Thus, the choice of representation in Definition 2.3

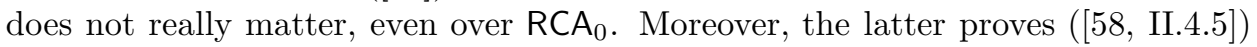
that the real number system satisfies all the axioms of an Archimedian ordered field, i.e. we generally work with the latter axiomatic properties in RM, rather than with the representations (whatever they are).

Finally, we mention the ECF-interpretation, as it will be needed below.

Remark 2.5. The technical definition of the ECF-interpretation may be found in [66. p. 138, 2.6]. Intuitively speaking, the ECF-interpretation $[A]_{\mathrm{ECF}}$ of a formula $A \in \mathrm{L}_{\omega}$ is just $A$ with all variables of type two and higher replaced by countable representations of continuous functionals. The ECF-interpretation connects $\mathrm{RCA}_{0}^{\omega}$ and $\mathrm{RCA}_{0}$ (See [34, Prop. 3.1]) in that if $\mathrm{RCA}_{0}^{\omega}$ proves $A$, then $\mathrm{RCA}_{0}$ proves $[A]_{\mathrm{ECF}}$,

\footnotetext{
${ }^{2}$ The 'early' constructions due to Dedekind (see e.g. [16]; using cuts) and Cantor (see e.g. [14]; using Cauchy sequences) were both originally published in 1872 .
} 
again 'up to language', as $\mathrm{RCA}_{0}$ is formulated using sets, and $[A]_{\mathrm{ECF}}$ is formulated using types, namely only using type zero and one objects. Note that for $A \in \mathrm{L}_{2}$, we have that $[A]_{\mathrm{ECF}}$ is just $A$ by definition.

2.2. Some axioms of higher-order arithmetic. We introduce some functionals which constitute the counterparts of $Z_{2}$, and some of the Big Five systems, in higher-order RM. We use the formulation of these functionals as in 34 .

First of all, $\mathrm{ACA}_{0}$ is readily derived from the following 'Turing jump' functional:

$$
\left(\exists \varphi^{2} \leq_{2} 1\right)\left(\forall f^{1}\right)[(\exists n)(f(n)=0) \leftrightarrow \varphi(f)=0] .
$$

and $\mathrm{ACA}_{0}^{\omega} \equiv \mathrm{RCA}_{0}^{\omega}+\left(\exists^{2}\right)$ proves the same sentences as $\mathrm{ACA}_{0}$ by [29, Theorem 2.5]. This functional is discontinuous at $f={ }_{1} 11 \ldots$, and $\left(\exists^{2}\right)$ is equivalent to the existence of $F: \mathbb{R} \rightarrow \mathbb{R}$ such that $F(x)=1$ if $x>_{\mathbb{R}} 0$, and 0 otherwise $([34, \S 3])$.

Secondly, $\Pi_{1}^{1}-\mathrm{CA}_{0}$ is readily derived from the following 'Suslin functional':

$$
\left(\exists S^{2} \leq_{2} 1\right)\left(\forall f^{1}\right)\left[\left(\exists g^{1}\right)\left(\forall x^{0}\right)(f(\bar{g} n)=0) \leftrightarrow S(f)=0\right],
$$

and $\Pi_{1}^{1}-\mathrm{CA}_{0}^{\omega} \equiv \mathrm{RCA}_{0}^{\omega}+\left(S^{2}\right)$ proves the same $\Pi_{3}^{1}$-sentences as $\Pi_{1}^{1}-\mathrm{CA}_{0}$ by [56. Theorem 2.2]. By definition, the Suslin functional $S^{2}$ can decide whether a $\Sigma_{1}^{1}$-formula (as in the left-hand side of $\left(S^{2}\right)$ ) is true or false. Note that we allow formulas with (type one) function parameters, but not with (higher type) functional parameters. The system $\Pi_{k}^{1}-C A_{0}^{\omega}$ is defined similarly via a functional $S_{k}^{2}$ deciding $\Sigma_{k}^{1}$-formulas. Thirdly, full second-order arithmetic $Z_{2}$ is readily derived from the sentence:

$$
\left(\exists E^{3} \leq_{3} 1\right)\left(\forall Y^{2}\right)\left[\left(\exists f^{1}\right) Y(f)=0 \leftrightarrow E(Y)=0\right],
$$

and we define $Z_{2}^{\Omega} \equiv R C A_{0}^{\omega}+\left(\exists^{3}\right)$, a conservative extension of $Z_{2}$ by [29, Cor. 2.6]. The (unique) functional from $\left(\exists^{3}\right)$ is also called ' $\exists^{3}$ ', and we will use a similar convention for other functionals.

Fourth, weak König's lemma 3 (WKL hereafter) easily follows from both the 'intuitionistic' and 'classical' fan functional, which are defined as follows:

$$
\begin{aligned}
& \left(\exists \Omega^{3}\right)\left(\forall Y^{2}\right)(\forall f, g \in C)(\bar{f} \Omega(Y)=\bar{g} \Omega(Y) \rightarrow Y(f)=Y(g)), \\
& \left(\exists \Phi^{3}\right)\left(\forall Y^{2} \in \text { cont }\right)(\forall f, g \in C)(\bar{f} \Phi(Y)=\bar{g} \Phi(Y) \rightarrow Y(f)=Y(g)),
\end{aligned}
$$

where ' $Y^{2} \in$ cont' means that $Y$ is continuous on Baire space $\mathbb{N}^{\mathbb{N}}$. Clearly, $\exists^{2}, S^{2}$, and $\exists^{3}$ are a kind of comprehension axiom. As it turns out, the comprehension for Cantor space functional also yields a conservative extension of $\mathrm{WKL}_{0}$ :

$$
\left(\exists \kappa_{0}^{3}\right)\left(\forall Y^{2}\right)\left[\kappa_{0}(Y)=0 \leftrightarrow(\exists f \in C)(Y(f)>0)\right],
$$

as MUC implies $\left(\kappa_{0}^{3}\right)$, and the former is conservative over $\mathrm{WKL}_{0}$ by [34, Cor. 3.15]. The subscript ' 0 ' in $\left(\kappa_{0}^{3}\right)$ has no purpose other than distinguishing this axiom from the related axiom $\left(\kappa^{3}\right)$ from [48.

Finally, recall that the Heine-Borel theorem (aka Cousin's lemma) states the existence of a finite sub-cover for an open cover of a compact space. Now, a functional $\Psi: \mathbb{R} \rightarrow \mathbb{R}^{+}$gives rise to the canonical cover $\cup_{x \in I} I_{x}^{\Psi}$ for $I \equiv[0,1]$, where $I_{x}^{\Psi}$ is the open interval $(x-\Psi(x), x+\Psi(x))$. Hence, the uncountable cover $\cup_{x \in I} I_{x}^{\Psi}$ has a finite sub-cover by the Heine-Borel theorem; in symbols:

$$
\left(\forall \Psi: \mathbb{R} \rightarrow \mathbb{R}^{+}\right)\left(\exists\left\langle y_{1}, \ldots, y_{k}\right\rangle\right)(\forall x \in I)(\exists i \leq k)\left(x \in I_{y_{i}}^{\Psi}\right) .
$$

\footnotetext{
${ }^{3}$ Note that we take ' $W K L$ ' to be the $\mathrm{L}_{2}$-sentence every infinite binary tree has a path as in [58], while the Big Five system $W K L_{0}$ is $R C A_{0}+W K L$, and $W K L_{0}^{\omega}$ is $R C A_{0}^{\omega}+W K L$.
} 
By the results in [50, 51], $Z_{2}^{\Omega}$ proves $\mathrm{HBU}$, but $\mathrm{Z}_{2}^{\omega} \equiv \cup_{k} \Pi_{k}^{1}-\mathrm{CA}_{0}^{\omega}$ cannot. The importance and naturalness of $\mathrm{HBU}$ is discussed in Section 4 .

Furthermore, since Cantor space (denoted $C$ or $2^{\mathbb{N}}$ ) is homeomorphic to a closed subset of $[0,1]$, the former inherits the same property. In particular, for any $G^{2}$, the corresponding 'canonical cover' of $2^{\mathbb{N}}$ is $\cup_{f \in 2^{\mathbb{N}}}[\bar{f} G(f)]$ where $\left[\sigma^{0^{*}}\right]$ is the set of all binary extensions of $\sigma$. By compactness, there is a finite sequence $\left\langle f_{0}, \ldots, f_{n}\right\rangle$ such that the set of $\cup_{i \leq n}\left[\bar{f}_{i} F\left(f_{i}\right)\right]$ still covers $2^{\mathbb{N}}$. By [50, Theorem 3.3], HBU is equivalent to the same compactness property for $C$, as follows:

$$
\left(\forall G^{2}\right)\left(\exists\left\langle f_{1}, \ldots, f_{k}\right\rangle\right)\left(\forall f^{1} \leq_{1} 1\right)(\exists i \leq k)\left(f \in\left[\overline{f_{i}} G\left(f_{i}\right)\right]\right) .
$$

Note that MUC implies $\mathrm{HBU}_{\mathrm{c}}$, i.e. the latter has weak first-order strength, but is extremely hard to prove by the aforementioned results.

Finally, we need a 'trivially uniform' version of ATR :

$$
\left(\exists \Phi^{1 \rightarrow 1}\right)\left(\forall X^{1}, f^{1}\right)\left[\mathrm{WO}(X) \rightarrow H_{f}(X, \Phi(X, f))\right],
$$

where $\mathrm{WO}(X)$ expresses that $X$ is a countable well-ordering and $H_{\theta}(X, Y)$ expresses that $Y$ is the result from iterating $\theta$ along $X$ (See [58, V] for details), and where $H_{f}(X, Y)$ is just $H_{\theta}(X, Y)$ with $\theta(n, Z)$ defined as $\left(\exists m^{0}\right)(f(n, m, \bar{Z} m)=0)$.

\section{MAIN RESUlts}

Our motivation and starting point is the splitting $\left(\exists^{3}\right) \leftrightarrow\left[\left(\kappa_{0}^{3}\right) \wedge\left(\exists^{2}\right)\right]$ communicated to us by Kohlenbach 4 (See [48, Rem. 6.13]). It is then a natural question if $\left(\kappa_{0}^{3}\right)$ can be split further, as discussed in Section 3.1. We obtain similar results for MUC in Section 3.2, which yields splittings and disjunctions for $\left(\exists^{2}\right),\left(\exists^{3}\right),\left(Z^{3}\right)$, and FF in Section 3.3. We similarly study HBU in Section 3.4, while other covering theorems, including the original Lindelöf lemma, are discussed in Section 3.5. As done in e.g. [27, we shall always write ' $A+B$ ' in the stead of ' $A \wedge B$ '.

3.1. Comprehension on Cantor space. We show that $\left(\kappa_{0}^{3}\right)$ defined as follows:

$$
\left(\exists \kappa_{0}^{3}\right)\left(\forall Y^{2}\right)\left[\kappa_{0}(Y)=0 \leftrightarrow(\exists f \in C)(Y(f)>0)\right],
$$

splits into the classical fan functional, given by $\mathrm{FF}$ as follows:

$$
\left(\exists \Phi^{3}\right)\left(\forall Y^{2} \in \text { cont }\right)(\forall f, g \in C)(\bar{f} \Phi(Y)=\bar{g} \Phi(Y) \rightarrow Y(f)=Y(g)),
$$

and a functional which tests for continuity on $\mathbb{N}^{\mathbb{N}}$, as follows:

$$
\left(\exists Z^{3}\right)\left(\forall Y^{2}\right)\left[Z(Y)=0 \leftrightarrow\left(\forall f^{1}\right)\left(\exists N^{0}\right)\left(\forall g^{1}\right)(\bar{f} N=\bar{g} N \rightarrow Y(f)=Y(g))\right] .
$$

We will tacitly use $\left(\exists^{2}\right) \rightarrow$ FF $\rightarrow$ WKL, which holds over RCA ${ }_{0}^{\omega}$ by [35, Prop. 4.10].

Theorem 3.1. The system $\mathrm{WKL}_{0}^{\omega}+\mathrm{QF}-\mathrm{AC}^{2,0}$ proves $\left(\kappa_{0}^{3}\right) \leftrightarrow\left[\left(Z^{3}\right)+\mathrm{FF}\right]$.

Proof. For the forward implication, we work in $\mathrm{WKL}_{0}^{\omega}+\mathrm{QF}-\mathrm{AC}^{2,0}+\left(\kappa_{0}^{3}\right)$. In case $\left(\exists^{2}\right)$ holds, we also have $\left(\exists^{3}\right)$, and the latter functional readily implies $\left(Z^{3}\right)$ and FF. In case of $\neg\left(\exists^{2}\right)$, all functionals $Y^{2}$ are continuous on Baire space by [34, Prop. 3.7], and $Z_{0}={ }_{3} 0$ is as required for $\left(Z^{3}\right)$. By WKL (and [35, Prop. 4.10]), all functionals $Y^{2}$ are uniformly continuous on Cantor space, i.e.

$$
\left(\forall Y^{2}\right)\left(\exists N^{0}\right) \underline{\left(\forall f^{1}, g^{1} \in C\right)(\bar{f} N=\bar{g} N \rightarrow Y(f)=Y(g)),}
$$

\footnotetext{
${ }^{4}$ The proof amounts to the observation that $\mathbb{N}^{\mathbb{N}}$ is recursively homeomorphic to a $\Pi_{2}^{0}$-subset of Cantor space. Since this set is computable in $\exists^{2}$, any oracle call to $\exists^{3}$ can be rewritten to an equivalent oracle call to $\kappa_{0}^{3}$, in a uniform way.
} 
and the underlined formula may be treated as quantifier-free by $\left(\kappa_{0}^{3}\right)$. Applying QF-AC ${ }^{2,0}$, we obtain FF. The law of excluded middle finishes this implication.

For the reverse implication, we work in $\operatorname{RCA}_{0}^{\omega}+\left(Z^{3}\right)+$ FF. In case of $\neg\left(\exists^{2}\right)$, all functionals $Y^{2}$ are continuous on Baire space by [34, Prop. 3.7], and FF readily implies $\left(\kappa_{0}^{3}\right)$ by noting that the latter restricted to $Y^{2}$ uniformly continuous on $C$ is trivial. In case of $\left(\exists^{2}\right)$, let $Y_{0}$ be $Y$ on $C$, and zero otherwise. Now define $\kappa_{0}$ as follows: in case $Z\left(Y_{0}\right)=0, Y_{0}$ is continuous on Cantor space, and use FF to decide whether $(\exists f \in C)(Y(f)>0)$; in case $Z\left(Y_{0}\right) \neq 0$, then $(\exists f \in C)\left(Y_{0}(f)>0\right)$, and $\kappa_{0}(Y):=0$. The law of excluded middle finishes this implication.

Corollary 3.2. The system $\mathrm{RCA}_{0}^{\omega}+\mathrm{QF}-\mathrm{AC} \mathrm{C}^{2,0}$ proves $\left[\left(\kappa_{0}^{3}\right)+\mathrm{WKL}\right] \leftrightarrow\left[\left(Z^{3}\right)+\mathrm{FF}\right]$ and the system $\operatorname{RCA}_{0}^{\omega}$ proves $\left(\exists^{3}\right) \leftrightarrow\left[\left(\exists^{2}\right)+\left(Z^{3}\right)\right]$.

3.2. The intuitionistic fan functional. A hallmark of intuitionistic mathematics is Brouwer's continuity theorem which expresses that all functions on the unit interval are (uniformly) continuous (10]). In the same vein, the intuitionistic fan functional $\Omega^{3}$ as in MUC provides a modulus of uniform continuity on Cantor space:

$$
\left(\exists \Omega^{3}\right)\left(\forall Y^{2}\right)(\forall f, g \in C)(\bar{f} \Omega(Y)=\bar{g} \Omega(Y) \rightarrow Y(f)=Y(g)) .
$$

This axiom can be split nicely into classical and non-classical parts as follows.

Theorem 3.3. The system $\mathrm{RCA}_{0}^{\omega}+\mathrm{QF}-\mathrm{AC}^{2,0}$ proves

$$
\mathrm{MUC} \leftrightarrow\left[\left(\kappa_{0}^{3}\right)+\mathrm{WKL}+\neg\left(\exists^{2}\right)\right] \leftrightarrow\left[\left(\kappa_{0}^{3}\right)+\mathrm{WKL}+\neg\left(S^{2}\right)\right] \leftrightarrow\left[\left(\kappa_{0}^{3}\right)+\mathrm{WKL}+\neg\left(\exists^{3}\right)\right] .
$$

Proof. For the first equivalence, assume MUC and note that the latter reduces the decision procedure for $(\exists f \in C)(Y(f)>0)$ to a finite search involving only $2^{\Omega(Y)}$ sequences. Furthermore, $\left(\exists^{2}\right)$ clearly implies the existence of a discontinuous function on Cantor space, i.e. MUC $\rightarrow \neg\left(\exists^{2}\right)$ follows, while MUC $\rightarrow$ WKL follows from [58, IV.2.3]. Now assume $\left(\kappa_{0}^{3}\right)+\mathrm{WKL}+\neg\left(\exists^{2}\right)$ and recall that by the latter all functionals $Y^{2}$ are continuous on Baire space by [34, Prop. 3.7]. By WKL (and [35. Prop. 4.10]), all functionals $Y^{2}$ are uniformly continuous on Cantor space, i.e.

$$
\left(\forall Y^{2}\right)\left(\exists N^{0}\right) \underline{\left(\forall f^{1}, g^{1} \in C\right)(\bar{f} N=\bar{g} N \rightarrow Y(f)=Y(g)),}
$$

and the underlined formula may be treated as quantifier-free by $\left(\kappa_{0}^{3}\right)$. Applying QF-AC $C^{2,0}$, we obtain MUC. For the remaining equivalences, since $\left(\exists^{3}\right) \leftrightarrow\left[\left(\kappa_{0}^{3}\right)+\right.$ $\left.\left(\exists^{2}\right)\right]$, we have that $\left[\neg\left(\exists^{3}\right)+\left(\kappa_{0}^{3}\right)\right] \rightarrow \neg\left(\exists^{2}\right)$, and the same for $\neg\left(S^{2}\right)$. Finally, note that $\left(\exists^{3}\right) \rightarrow\left(S^{2}\right) \rightarrow\left(\exists^{2}\right)$ implies $\neg\left(\exists^{2}\right) \rightarrow \neg\left(S^{2}\right) \rightarrow \neg\left(\exists^{3}\right)$.

Recall the ECF-interpretation introduced at the end of Section 2.1. By [37, §9.5], we have $[\mathrm{MUC}]_{\mathrm{ECF}} \leftrightarrow \mathrm{WKL}$ and $\mathrm{WKL} \rightarrow\left[\left(\kappa_{0}^{3}\right)\right]_{\mathrm{ECF}}$, while $\left[\left(\exists^{2}\right)\right]_{\mathrm{ECF}} \leftrightarrow(0=1)$ as $\exists^{2}$ is discontinuous (and therefore has no countable representation). Hence, $\neg\left(\exists^{2}\right)$ cannot be replaced by $\neg \mathrm{ACA}_{0}$ in the theorem, as $[A]_{\mathrm{ECF}} \leftrightarrow A$ for $A \in \mathrm{L}_{2}$.

Furthermore, the axiom MUC can also be split as follows. As an exercise, the reader should show that the corollary also goes through for $\mathrm{RCA}_{0}^{\omega}$.

Corollary 3.4. The system $\mathrm{RCA}_{0}^{\omega}+\mathrm{QF}-\mathrm{AC}^{2,0}$ proves

$$
\mathrm{MUC} \leftrightarrow\left[\mathrm{FF}+\neg\left(\exists^{2}\right)\right] \leftrightarrow\left[\mathrm{FF}+\left(Z^{3}\right)+\neg\left(S^{2}\right)\right] \leftrightarrow\left[\mathrm{FF}+\left(Z^{3}\right)+\neg\left(\exists^{3}\right)\right] .
$$

Proof. By Corollary 3.2 and the theorem, we have MUC $\leftrightarrow\left[\left(Z^{3}\right)+\mathrm{FF}+\neg\left(\exists^{2}\right)\right]$, and we may omit $\left(Z^{3}\right)$ because all functionals on $\mathbb{N}^{\mathbb{N}}$ are continuous given $\neg\left(\exists^{2}\right)$. By the same corollary, $\left[\left(Z^{3}\right)+\mathrm{FF}+\neg\left(S^{2}\right)\right] \leftrightarrow\left[\left(\kappa_{0}^{3}\right)+\mathrm{WKL}+\neg\left(S^{2}\right)\right]$, and the latter is equivalent to MUC by the theorem. The same reasoning applies to $\neg\left(\exists^{3}\right)$. 
As a result of the previous, the $\mathrm{RM}$ of $\left(\kappa_{0}^{3}\right)$ is pretty robust. Indeed, for a sentence W implying $\left(\kappa_{0}^{3}\right)$, if the former implies the existence of a discontinuous functional, we obtain $\left(\exists^{3}\right)$ by [34, §3]. What happens when $\mathrm{W}$ does not imply this existence, is captured (in part) by the following theorem.

Theorem 3.5. If $\mathrm{MUC} \rightarrow \mathrm{W}$ and $\left(\exists^{3}\right) \rightarrow \mathrm{W} \rightarrow\left(\kappa_{0}^{3}\right)$ over $\mathrm{RCA}_{0}^{\omega}$, then $\mathrm{WKL}_{0}^{\omega}+$ QF-AC ${ }^{2,0}$ proves $\mathrm{W} \leftrightarrow\left(\kappa_{0}^{3}\right)$.

Proof. The forward implication is immediate. For the reverse implication, consider $\left(\exists^{2}\right) \vee \neg\left(\exists^{2}\right)$; in the former case, we obtain $\left(\exists^{3}\right)$ and hence $\mathbf{W}$, while in the latter case, we may use the proof of Theorem 3.3. the continuity of all functionals on Baire space and WKL imply (3.1), which yields MUC thanks to $\left(\kappa_{0}^{3}\right)$ and QF-AC ${ }^{2,0}$, and $\mathrm{W}$ follows by assumption.

3.3. More splittings and disjunctions. The results regarding the non-classical axiom MUC also yield splittings for the classical axioms FF, $\left(\exists^{2}\right),\left(\exists^{3}\right)$, and $\left(Z^{3}\right)$.

Theorem 3.6. The system $\mathrm{RCA}_{0}^{\omega}+\mathrm{QF}-\mathrm{AC}^{2,0}$ proves

$$
\left[\left(\kappa_{0}^{3}\right)+\mathrm{WKL}\right] \leftrightarrow\left[\left(\exists^{3}\right) \vee \mathrm{MUC}\right] \leftrightarrow\left[\left(\kappa_{0}^{3}\right)+\mathrm{FF}\right]
$$

$\mathrm{RCA}_{0}^{\omega}$ proves $\mathrm{FF} \leftrightarrow\left[\left(\exists^{2}\right) \vee \mathrm{MUC}\right]$, while $\mathrm{WKL} \mathrm{L}_{0}^{\omega}+\mathrm{QF}-\mathrm{AC}^{2,0}$ proves $\mathrm{FF} \leftrightarrow\left[\left(\exists^{2}\right) \vee\left(\kappa_{0}^{3}\right)\right]$.

Proof. For the first equivalence in (3.2), the reverse implication is immediate if $\left(\exists^{3}\right)$ holds, while it follows from Theorem 3.3 if MUC holds. For the forward implication, if $\left(\exists^{2}\right)$, we have $\left(\exists^{3}\right)$, while if $\neg\left(\exists^{2}\right)$, we follow the proof of Theorem 3.3 to obtain MUC. The second equivalence in (3.2) follows in the same way. For the third equivalence, the reverse implication is immediate, while the forward implication follows by considering $\left(\exists^{2}\right) \vee \neg\left(\exists^{2}\right)$, noting that all functionals on $C$ are continuous in the latter case. For the final equivalence, we only need to prove $\left(\kappa_{0}^{3}\right) \rightarrow$ FF given WKL. The implication is immediate if $\left(\exists^{2}\right)$, while it follows in the same way as in the proof of (3.2) in case $\neg\left(\exists^{2}\right)$.

Theorem 3.7. The system $\mathrm{RCA}_{0}^{\omega}+\mathrm{QF}-\mathrm{AC} \mathrm{C}^{2,0}$ proves $\left(\exists^{2}\right) \leftrightarrow[\mathrm{FF}+\neg \mathrm{MUC}]$ and $\left(\exists^{3}\right) \leftrightarrow\left[\mathrm{FF}+\left(Z^{3}\right)+\neg \mathrm{MUC}\right]$ and $\left(Z^{3}\right) \leftrightarrow\left[\left(\exists^{3}\right) \vee \neg\left(\exists^{2}\right)\right] \leftrightarrow\left[\left(\exists^{3}\right) \vee \neg \mathrm{FF} \vee \mathrm{MUC}\right]$.

Proof. The second equivalence follows from the first one by Corollary 3.2 For the first equivalence, the forward implication is immediate, and for the reverse implication, Corollary 3.4 implies $\neg$ MUC $\leftrightarrow\left[\neg F F \vee\left(\exists^{2}\right)\right]$. Since FF is assumed, we obtain $\left(\exists^{2}\right)$. For the third equivalence, the reverse implication is immediate in case $\left(\exists^{3}\right)$, while $Z={ }_{3} 0$ works if $\neg\left(\exists^{2}\right)$ as all functionals on Baire space are continuous then; for the forward implication, consider $\left(\exists^{2}\right) \vee \neg\left(\exists^{2}\right)$ and use Corollary 3.2 in the former case. The final equivalence now follows from the first equivalence.

3.4. Heine-Borel compactness. We discuss the rich world of splittings and disjunctions associated to Heine-Borel compactness as in $\mathrm{HBU}$, which we recall:

$$
\left(\forall \Psi: \mathbb{R} \rightarrow \mathbb{R}^{+}\right)\left(\exists\left\langle y_{1}, \ldots, y_{k}\right\rangle\right)(\forall x \in I)(\exists i \leq k)\left(x \in I_{y_{i}}^{\Psi}\right) .
$$

Note that $\mathrm{HBU}_{\mathrm{c}}$ similarly expresses the open-cover compactness of Cantor space. First of all, we establish a nice disjunction for WKL.

Theorem 3.8. The system $\mathrm{RCA}_{0}^{\omega}$ proves that

$$
\mathrm{WKL} \leftrightarrow\left[\left(\exists^{2}\right) \vee \mathrm{HBU}_{\mathrm{c}}\right] \leftrightarrow[\mathrm{X} \vee \mathrm{HBU}] \leftrightarrow\left[\mathrm{FF} \vee \mathrm{HBU}_{\mathrm{c}}\right]
$$

for any $\mathrm{X} \in \mathrm{L}_{2}$ such that $\mathrm{ACA}_{0} \rightarrow \mathrm{X} \rightarrow \mathrm{WKL}_{0}$. 
Proof. We prove the first equivalence and note that the other equivalences in (3.3) follow in the same way. The reverse implication follows from $\mathrm{HBU}_{\mathrm{c}} \rightarrow \mathrm{WKL}$ and $\left(\exists^{2}\right) \rightarrow \mathrm{ACA}_{0} \rightarrow \mathrm{WKL}_{0}$. For the forward implication, note that all functionals on $\mathbb{N}^{\mathbb{N}}$ are continuous given $\neg\left(\exists^{2}\right)$, and hence uniformly continuous on $C$ by WKL. Hence, all functionals on $C$ have an upper bound, which immediately implies $\mathrm{HBU}_{\mathrm{c}}$. The law of excluded middle $\left(\exists^{2}\right) \vee \neg\left(\exists^{2}\right)$ finishes the proof.

As noted in Section 2.2, the systems $\mathrm{ACA}_{0}^{\omega}$ and $\Pi_{1}^{1}-\mathrm{CA}_{0}^{\omega}$ are conservative extensions of their second-order counterparts. However, the ECF-translation leaves $\mathrm{L}_{2}$-sentences unchanged, while translating $\left(\exists^{2}\right)$ to ' $0=1$ '. As a result, the disjuncts in the first equivalence in (3.4) below are independent.

Corollary 3.9. The system $\mathrm{RCA}_{0}^{\omega}$ proves that

$$
\mathrm{WKL} \leftrightarrow\left[\Pi_{1}^{1}-\mathrm{CA}_{0} \vee\left(\exists^{2}\right) \vee \mathrm{HBU}_{\mathrm{c}}\right] \leftrightarrow[\mathrm{X} \vee \mathrm{FF} \vee \mathrm{HBU}] .
$$

for any $\mathrm{X} \in \mathrm{L}_{2}$ such that $\mathrm{X} \rightarrow \mathrm{WKL}_{0}$.

Secondly, let $\mathrm{T}_{1}$ be [21, Theorem 4.5.2] i.e. the $\mathrm{L}_{2}$-sentence: For all $k \in \mathbb{N}$ and all compact metric spaces $X$ and continuous functions $F: X \rightarrow X, F^{k}$ is a continuous

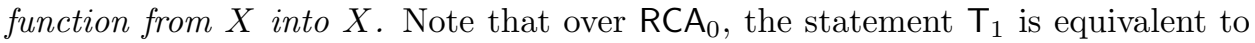
WKL $\vee \Sigma_{2}^{0}$-IND, where the latter is the induction schema restricted to $\Sigma_{2}^{0}$-formulas.

Corollary 3.10. The system $\mathrm{RCA}_{0}^{\omega}$ proves

$$
\mathrm{T}_{1} \leftrightarrow\left[\mathrm{HBU} \vee \Sigma_{2}^{0}-\mathrm{IND}\right] \leftrightarrow\left[\mathrm{WKL} \vee \Sigma_{2}^{0}-\mathrm{IND}\right] \leftrightarrow\left[\mathrm{FF} \vee \mathrm{HBU} \vee \Sigma_{2}^{0}-\mathrm{IND}\right] .
$$

Proof. We only need to prove the first equivalence. The reverse direction is immediate as $\mathrm{HBU} \rightarrow \mathrm{WKL} \rightarrow \mathrm{T}_{1}$ and $\Sigma_{2}^{0}$-IND $\rightarrow \mathrm{T}_{1}$. For the forward direction,

$$
\mathrm{T}_{1} \rightarrow\left[\mathrm{WKL} \vee \Sigma_{2}^{0} \text {-IND }\right] \rightarrow\left[\mathrm{ACA}_{0} \vee \mathrm{HBU} \vee \Sigma_{2}^{0} \text {-IND }\right]
$$

since $\mathrm{ACA}_{0}$ implies $\Sigma_{k}^{0}$-IND (for any $k$ ), and we obtain the equivalence in (3.5)).

We provide another proof of the forward direction that will be useful for Section 4. Assume $\mathrm{T}_{1}$ and consider $\Sigma_{2}^{0}$-IND $\vee \neg\left[\Sigma_{2}^{0}\right.$-IND]. In the erstwhile case, we are done. In the latter case, we must have WKL due to $\mathrm{T}_{1} \leftrightarrow\left[\mathrm{WKL} \vee \Sigma_{2}^{0}\right.$-IND]; since $\left(\exists^{2}\right) \rightarrow$ ACA $_{0} \rightarrow \Sigma_{2}^{0}$-IND, we also obtain $\neg\left(\exists^{2}\right)$, and hence HBU as in the proof of the theorem, and we are done.

Thirdly, while (3.3) and (3.5) may come across as spielerei, WKL $\leftrightarrow\left[\mathrm{ACA}_{0} \vee \mathrm{HBU}\right]$ is actually of great conceptual importance, as follows.

Template 3.11. To prove a theorem $\mathrm{T}$ in $\mathrm{WKL} \mathrm{L}_{0}^{\omega}$, proceed as follows:

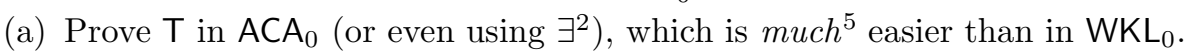

(b) Prove $\mathrm{T}$ in $\mathrm{RCA}_{0}^{\omega}+\mathrm{HBU}$ using the existing 'uniform' proof from the literature based on Cousin's lemma (See e.g. [3,4,7, 24, 31, 60, 65]).

(c) Conclude from (a) and (b) that $\mathrm{T}$ can be proved in $\mathrm{WKL}_{0}^{\omega}$.

Hence, even though the goal of RM is to find the minimal axioms needed to prove a theorem, one can nonetheless achieve this goal by (only) using non-minimal axioms. We leave it to the reader to ponder how much time and effort could have

\footnotetext{
${ }^{5}$ For instance, the functional $\exists^{2}$ uniformly converts between binary-represented reals and realsas-Cauchy-sequences. In this way, one need not worry about representations and the associated extensionality like in Definition 2.3 (5). By the proof of [35. Prop. 4.7], $\exists^{2}$ also uniformly converts a continuous function into an RM-code, i.e. we may 'recycle' proofs in second-order arithmetic.
} 
been (and will be) saved using the previous three steps (for WKL or other axioms). As an exercise, the reader should try to prove Pincherle's theorem ([38, p. 97]) via Template 3.11, using realisers for the antecedent as in the original [54. The former theorem is studied in [51, where Template 3.11 is used frequently.

Fourth, in [8, Theorem 2.28], an equivalence between $\neg \mathrm{WKL}_{0} \vee \mathrm{ACA}_{0}$ and the following theorem is established: there is a complete theory with a non-principal type and only finitely many models up to isomorphism. The contraposition of the latter, which we shall denote $T_{0}$ and satisfies $T_{0} \leftrightarrow \mathrm{WKL}_{0}+\neg \mathrm{ACA}_{0}$, is described in [8] as a peculiar but natural statement about some pre-ordering.

Corollary 3.12. The system $\mathrm{RCA} A_{0}^{\omega}+\mathrm{T}_{0}$ proves $\mathrm{HBU}$. The system $\mathrm{RCA}_{0}^{\omega}$ proves $\mathrm{T}_{0} \leftrightarrow\left[\mathrm{WKL}_{0}+\neg \mathrm{ACA}_{0}\right] \leftrightarrow\left[\mathrm{HBU}+\neg \mathrm{ACA}_{0}\right]$

Proof. In $\neg \mathrm{T}_{0} \leftrightarrow\left[\neg \mathrm{WKL}_{0} \vee A C A_{0}\right]$, use (3.3) to replace $\mathrm{WKL}$ by $\mathrm{ACA}_{0} \vee \mathrm{HBU}$, i.e.

$$
\neg \mathrm{T}_{0} \leftrightarrow\left[\left[\neg \mathrm{ACA}_{0}+\neg \mathrm{HBU}\right] \vee \mathrm{ACA} \mathrm{A}_{0}\right] \leftrightarrow\left[\underline{\left[\left[\mathrm{ACA}_{0} \vee \mathrm{ACA}_{0}\right]\right.}+\left[\mathrm{ACA}_{0} \vee \neg \mathrm{HBU}\right]\right] .
$$

Omitting the underlined formula, the second (and first) part follows.

We provide another proof of $\mathrm{T}_{0} \rightarrow\left[\mathrm{HBU}+\neg \mathrm{ACA}_{0}\right]$ that will be useful for Section 4. Since $\mathrm{T}_{0} \rightarrow\left[\mathrm{WKL}_{0}+\neg \mathrm{ACA}_{0}\right]$, we also have $T_{0} \rightarrow\left[\mathrm{WKL}_{0}+\neg\left(\exists^{2}\right)\right]$, and $\mathrm{HBU}$ follows as in the proof of the theorem.

By the previous, the negation of $\mathrm{WKL}_{0}$ or $\mathrm{ACA}_{0}$ implies axioms of Brouwer's intuitionistic mathematics, i.e. strange (as in 'non-classical') behaviour is almost guaranteed. The equivalence involving $\neg W_{K} L_{0} \vee A C A_{0}$ remains surprising. By contrast, $T_{0}$ seems fairly normal, relative to e.g. $T_{1}$, by the following result.

Corollary 3.13. The system $\mathrm{RCA}_{0}$ proves $\mathrm{T}_{1} \leftrightarrow\left(\mathrm{T}_{0} \vee \Sigma_{2}^{0}\right.$-IND), $\mathrm{WKL}_{0} \leftrightarrow\left[\mathrm{ACA}_{0} \vee\right.$ $\left.\mathrm{T}_{0}\right]$, and $\left(\mathrm{T}_{0} \vee \mathrm{T}_{1}\right) \leftrightarrow \mathrm{T}_{1}$.

Proof. The second forward implication follows from $\mathrm{ACA}_{0} \vee \neg \mathrm{ACA}_{0}$, while the second reverse implication is immediate. The first reverse implication is immediate, while the first forward implication follows from:

$$
\mathrm{T}_{1} \rightarrow\left[\mathrm{WKL}_{0} \vee \Sigma_{2}^{0}-\mathrm{IND}\right] \rightarrow\left[\mathrm{ACA}_{0} \vee \mathrm{T}_{0} \vee \Sigma_{2}^{0}-\mathrm{IND}\right] \rightarrow\left[\mathrm{T}_{0} \vee \Sigma_{2}^{0}-\mathrm{IND}\right]
$$

since $\mathrm{ACA}_{0}$ proves induction for any arithmetical formula. The third equivalence follows by considering all cases in the disjunction that is $T_{1}$.

Similar to Corollary 3.9. Theorem 3.6 has the following corollary. Note that the ECF-translation again implies the independence of the disjuncts in (3.6), except that we do not know whether $\mathrm{T}_{0} \rightarrow$ MUC, over say $\mathrm{RCA}_{0}^{\omega}$.

Corollary 3.14. The system $\mathrm{RCA}_{0}^{\omega}+\mathrm{QF}-\mathrm{AC}^{2,0}+\mathrm{FF}$ proves

$$
\left[\left(\exists^{3}\right) \vee \mathrm{MUC}\right] \leftrightarrow\left(\kappa_{0}^{3}\right) \leftrightarrow\left[\left(\exists^{3}\right) \vee \mathrm{MUC} \vee \mathrm{T}_{0}\right] .
$$

Proof. The first equivalence and the second forward direction is immediate in light of (3.2). For the second reverse direction, $T_{0}$ implies $W K L_{0}$ and $\neg A C A_{0}$ by definition. The latter implies $\neg\left(\exists^{2}\right)$, i.e. all functions on Cantor space are continuous, and the fan functional as in FF readily yields $\left(\kappa_{0}^{3}\right)$.

Finally, the negation of HBU also occurs naturally as follows, where we recall:

$$
\left(\exists \Phi^{1 \rightarrow 1}\right)\left(\forall X^{1}, f^{1}\right)\left[\mathrm{WO}(X) \rightarrow H_{f}(X, \Phi(X, f))\right],
$$

Theorem 3.15. The system $\mathrm{RCA} A_{0}^{\omega}+\mathrm{FF}+\mathrm{QF}-\mathrm{AC}^{2,1}$ proves $\left(\exists^{2}\right) \leftrightarrow[\mathrm{UATR} \vee \neg \mathrm{HBU}]$. 
Proof. For the forward implication, consider HBU $\vee \neg \mathrm{HBU}$. In the former case, we obtain UATR by [48, Cor. 6.6] and [50, Theorem 3.3]. For the reverse implication, $[\neg \mathrm{HBU}+\mathrm{FF}] \rightarrow\left(\exists^{2}\right)$, which follows from MUC $\rightarrow \mathrm{HBU}$ and Theorem 3.7

Remark 3.16. It is a natural RM-question, posed previously by Hirschfeldt (see [43, §6.1]), whether the extra axioms are needed in the base theory of Theorem 3.15. The answer is positive in this case: the ECF-translation converts the equivalence in the theorem to $(0=1) \leftrightarrow[(0=1) \vee \neg W K L]$, which is only true if WKL (which is exactly $\left.[\mathrm{FF}]_{\mathrm{ECF}}\right)$ is in the base theory. Hence, the base theory needs WKL.

The above results, (3.3) and (3.5) in particular, suggests that mathematical naturalness does not inherit to disjuncts, which is in accordance with our intuitions.

3.5. Other covering theorems. We study two covering lemmas related to HBU, namely the Lindelöf lemma and a weak version of HBU.

3.5.1. The Lindelöf lemma. We study splittings and disjunctions for the Lindelöf lemma LIN from [50]. We stress that our formulation of HBU and LIN is faithful to the original theorems from 1895 and 1903 by Cousin ([15]) and Lindelöf ([36]).

Definition 3.17. [LIN] For every $\Psi: \mathbb{R} \rightarrow \mathbb{R}^{+}$, there is a sequence of open intervals $\cup_{n \in \mathbb{N}}\left(a_{n}, b_{n}\right)$ covering $\mathbb{R}$ such that $(\forall n \in \mathbb{N})(\exists x \in \mathbb{R})\left[\left(a_{n}, b_{n}\right)=I_{x}^{\Psi}\right]$.

The final result in the following theorem should be compared to (3.3).

Theorem 3.18. Let $\mathrm{X} \in \mathrm{L}_{2}$ be such that $\mathrm{ACA}_{0} \rightarrow \mathrm{X} \rightarrow \mathrm{WKL}_{0}$.

(a) The system $\mathrm{RCA}_{0}^{\omega}+\mathrm{QF}-\mathrm{AC}^{0,1}$ proves $\mathrm{LIN} \leftrightarrow[\mathrm{HBU} \vee \neg \mathrm{WKL}] \leftrightarrow[\mathrm{HBU} \vee \neg \mathrm{X}]$.

(b) If $\mathrm{Y} \in \mathrm{L}_{2}$ is provable in $\mathrm{ACA}_{0}$ but not in $\mathrm{RCA}_{0}$, then $\mathrm{RCA}_{0}^{\omega}$ proves $\mathrm{Y} \vee \mathrm{LIN}$, as well as $\Pi_{1}^{1}-\mathrm{CA}_{0} \vee\left(\exists^{2}\right) \vee \mathrm{LIN}$.

Proof. For the first item, RCA ${ }_{0}^{\omega}+$ QF-AC ${ }^{0,1}$ proves [LIN + WKL] $\leftrightarrow$ HBU by [50, Theorem 3.13]. Hence, the first forward implication follows from WKL $\vee \neg W K L$. For the first reverse implication, LIN follows from HBU by the aforementioned equivalence. In case $\neg$ WKL holds, we also have $\neg\left(\exists^{2}\right)$, as $(\exists)^{2} \rightarrow$ WKL. Hence, all functionals on $\mathbb{R}$ are continuous by [34, Prop. 3.12], and the countable sub-cover provided by the rationals suffices for the conclusion of LIN. The second equivalence follows in the same way by considering $X \vee \neg X$. For the second item, consider $\left(\exists^{2}\right) \vee \neg\left(\exists^{2}\right)$.

We now obtain a nice corollary to Theorems 3.15 and 3.18 In light of Remark 3.16. WKL also suffices for the base theory in the latter theorem.

Corollary 3.19. The system $\mathrm{WKL}_{0}^{\omega}+$ QF-AC ${ }^{2,1}$ proves $\left(\exists^{2}\right) \leftrightarrow[\mathrm{UATR} \vee \neg \mathrm{HBU}]$. The system $\mathrm{RCA}_{0}^{\omega}+$ QF-AC ${ }^{2,1}$ proves $\left(\exists^{2}\right) \leftrightarrow[$ UATR $\vee \neg \mathrm{LIN}]$.

Proof. The first forward implication follows as in the proof of Theorem 3.15, For the first reverse implication, the case $\neg \mathrm{HBU}$ implies $\left(\exists^{2}\right)$ by considering (3.8). The second equivalence now follows from the first item of Theorem 3.18

Finally, we discuss foundational implications of our results. Now, (3.3) implies:

$$
\neg \mathrm{LIN} \leftrightarrow[\mathrm{WKL}+\neg \mathrm{HBU}] \text { and } \neg \mathrm{LIN} \rightarrow\left(\exists^{2}\right) .
$$

On one hand, thanks to the ECF-translation, $W K L_{0}^{\omega}+\mathrm{HBU}$ is a conservative extension of $\mathrm{WKL}_{0}$, which in turn is a $\Pi_{2}^{0}$-conservative extension of primitive recursive 
arithmetic PRA. The latter is generally believed to correspond to Hilbert's finitistic mathematics (62]). Hence, following Simpson's remarks on finitistic mathematics ([58, IX.3.18]), WKL $L_{0}^{\omega}+\mathrm{HBU}$ also contributes to the partial realisation of Hilbert's program for the foundations of mathematics. On the other hand, $\mathrm{RCA}_{0}^{\omega}+\mathrm{WKL}+\neg \mathrm{HBU}$ and $\mathrm{RCA}_{0}^{\omega}+\mathrm{QF}-\mathrm{AC}^{0,1}+\neg \mathrm{LIN}$ imply $\left(\exists^{2}\right)$, i.e. these systems do not contribute to Hilbert's program in the aforementioned way.

Hence, if one values partial realisations of Hilbert program (which are called 'very important' by Simpson in [58, IX.3.18]), then HBU and LIN are practically forced upon one, in light of the previous. However, these covering lemmas require full second-order arithmetic as in $\mathrm{Z}_{2}^{\Omega}$ for a proof, i.e. they fall far outside of the Big Five classification of RM.

Finally, we consider the Lindelöf lemma for Baire space, denoted $\operatorname{LIN}\left(\mathbb{N}^{\mathbb{N}}\right)$ and studied in [50,51. Similar to Corollary 3.9 , one can prove the following equivalence:

$$
\left[\mathrm{WKL} \vee \operatorname{LIN}\left(\mathbb{N}^{\mathbb{N}}\right)\right] \leftrightarrow\left[\Pi_{1}^{1}-\mathrm{CA}_{0} \vee(\exists \Xi) \operatorname{LIN}(\Xi) \vee\left(\exists^{2}\right) \vee \mathrm{HBU}_{\mathrm{c}}\right]
$$

where $(\exists \Xi) \operatorname{LIN}(\Xi)$ states the existence of a functional $\Xi^{2 \rightarrow(0 \rightarrow 1)}$ that outputs the countable sub-cover from $\operatorname{LIN}\left(\mathbb{N}^{\mathbb{N}}\right)$.

3.5.2. Weak Heine-Borel compactness. We study WHBU, a weak version of HBU based on weak weak König's lemma (WWKL hereafter; see [58, X.1]). Note that WWKL is exceptional in that it is a theorem from the RM zoo that does sport a number of equivalences involving natural/mathematical statements.

In particular, by [58, X.1.9], WWKL is equivalent to the statement that any cover $\cup_{n \in \mathbb{N}}\left(a_{n}, b_{n}\right) \subset[0,1]$ is such that $\sum_{n=0}^{\infty}\left|a_{n}-b_{n}\right| \geq 1$, which is of independent6 historical interest. We define the higher-order version of this covering theorem as:

$$
\left(\forall \Psi: \mathbb{R} \rightarrow \mathbb{R}^{+}, k \in \mathbb{N}\right)\left(\exists\left\langle y_{1}, \ldots, y_{n}\right\rangle\right)\left(\sum_{i=1}^{n}\left|I_{y_{i}}^{\Psi}\right| \geq 1-\frac{1}{2^{k}}\right) .
$$

We could also use the statement $\mathrm{HBU}_{\mathrm{ml}}$ from [52, §3.3] instead of WHBU, but the latter is more elegant, and does not depend on the notion of randomness.

Theorem 3.20. Let $\mathrm{X} \in \mathrm{L}_{2}$ be such that $\mathrm{ACA}_{0} \rightarrow \mathrm{X} \rightarrow \mathrm{WWKL}_{0}$. $\mathrm{RCA}_{0}^{\omega}$ proves

$$
\mathrm{WWKL} \leftrightarrow\left[\left(\exists^{2}\right) \vee \mathrm{WHBU}\right] \leftrightarrow[\mathrm{X} \vee \mathrm{WHBU}] .
$$

Proof. This theorem is proved in the same way as Theorem 3.8. Indeed, for the first forward implication, consider $\left(\exists^{2}\right) \vee \neg\left(\exists^{2}\right)$ and note that in the latter case $\cup_{q \in[0,1] \cap \mathbb{Q}} I_{q}^{\Psi}$ is a countable sub-cover of the canonical cover since all functions are continuous. The first reverse implication is trivial in light of [58, X.1.9], and the other equivalences are proved similarly (and using Theorem 3.8).

Similar to Corollary 3.9, we have the following corollary.

Theorem 3.21. Let $\mathrm{X} \in \mathrm{L}_{2}$ be such that $\mathrm{X} \rightarrow \mathrm{WWKL}_{0}$. $\mathrm{RCA}_{0}^{\omega}$ proves

$$
\mathrm{WWKL} \leftrightarrow\left[\Pi_{1}^{1}-\mathrm{CA}_{0} \vee\left(\exists^{2}\right) \vee \mathrm{WHBU}\right] \leftrightarrow[\mathrm{X} \vee \mathrm{FF} \vee \mathrm{WHBU}] .
$$

One also readily proves that (3.8) can be extended to (3.8) $\leftrightarrow W W K L \vee \operatorname{LIN}\left(\mathbb{N}^{\mathbb{N}}\right)$.

The following version of Corollary 3.10 for WHBU is readily proved based on WWKL $\vee \Sigma_{2}^{0}$-IND and (3.9). We can prove similar results for the strong bounding principles and bounded comprehension principles instead of induction ([58, p. 72]).

\footnotetext{
${ }^{6}$ It is an interesting historical tidbit that a two-dimensional version of [58, X.1.9.3] was Borel's motivation for formulating and proving the (countable) Heine-Borel theorem ([11, p. 50, Note]).
} 
Corollary 3.22. The system $\mathrm{RCA}_{0}^{\omega}$ proves

$$
\left[\mathrm{WHBU} \vee \Sigma_{2}^{0}-\mathrm{IND}\right] \leftrightarrow\left[\mathrm{WWKL} \vee \Sigma_{2}^{0}-\mathrm{IND}\right] .
$$

We can also obtain a version of Theorem 3.18 for WHBU.

Corollary 3.23. Let $\mathrm{X} \in \mathrm{L}_{2}$ be such that $\mathrm{ACA}_{0} \rightarrow \mathrm{X} \rightarrow \mathrm{WWKL}_{0}$; the system $\mathrm{RCA}_{0}^{\omega}+\mathrm{QF}-\mathrm{AC}^{0,1}$ proves $\mathrm{LIN} \leftrightarrow[\mathrm{WHBU} \vee \neg \mathrm{WWKL}] \leftrightarrow[\mathrm{WHBU} \vee \neg \mathrm{X}]$.

Finally, let $(n+1)-W W K L$ be the generalisation of WWKL to trees computable in the $n$-th Turing jump, as formulated in [2]. Note that $\mathrm{ACA}_{0} \rightarrow(n+2)-\mathrm{WWKL} \rightarrow$

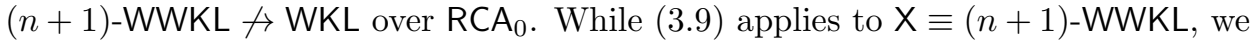
also have the following corollary.

Corollary 3.24. For $n \geq 1, \mathrm{RCA}_{0}^{\omega}$ proves $[\mathrm{HBU} \vee n$-WWKL $] \leftrightarrow[\mathrm{WKL} \vee n$-WWKL].

Proof. The forward implication is immediate, while for the reverse implication follows from (3.3) as WKL $\rightarrow\left[\mathrm{ACA}_{0} \vee \mathrm{HBU}\right] \rightarrow[n-\mathrm{WWKL} \vee \mathrm{HBU}]$.

Finally, it is a natural question if there are other theorems in the RM zoo for which we can find results like (3.3) and (3.9). We will provide a positive answer for (fragments of) Ramsey's theorem in a future publication.

\section{Foundational MUSings}

We provide an explanation for our results regarding splittings and disjunctions in Section 4.2, following an introduction in Section 4.1. The bigger picture is discussed in Sections 4.3 and 4.4 .

4.1. Introduction: continuity and discontinuity. In the below discussion, a central role is played by continuity. To be absolutely clear, our use of 'continuity' refers to the usual 'epsilon-delta' definition of functionals of type two or higher, unless stated otherwise. By [34, Prop. 3.7 and 3.12], the existence of a discontinuous, i.e. not everywhere continuous, functional is equivalent to $\left(\exists^{2}\right)$, for both $\mathbb{N}^{\mathbb{N}}$ and $\mathbb{R}$.

On one hand, it is well-known that $\mathrm{L}_{2}$ provides representations for discontinuous functions. For instance, measurable functions are represented by sequences of continuous functions in RM (see [58, X.1.11]). Furthermore, the basic theory of Borel functions and analytic sets can be developed in $\mathrm{ATR}_{0}$ via codes for Borel sets (see [58, V]). In other words, second-order arithmetic can model/represent discontinuous phenomena, and the purpose of this approach is to reconstitute these concepts in a way that accommodates their main applications within regions of the Gödel hierarchy (see [59]) that are as far down in the hierarchy as possible. For this kind of purpose, subsystems of $Z_{2}$ have been tremendously useful.

On the other, for $A \in \mathrm{L}_{2}$ provable in $\mathrm{Z}_{2}, \mathrm{RCA}_{0}^{\omega}+A$ cannot prove the existence of a discontinuous function, thank 7 to the ECF-translation and assuming $Z_{2}$ is consistent. Hence, second-order arithmetic can model/represent (certain) discontinuous phenomena, but it cannot prove (in the extended language $L_{\omega}$ ) the existence of e.g. a discontinuous function like $\exists^{2}$. Nonetheless, discontinuous functions entered the mathematical mainstream already around 1850 thanks to Riemann's Habilschrift:

\footnotetext{
${ }^{7}$ The ECF-translation of $\left(\exists^{2}\right)$ is ' $0=1$ ', while it does not change $A \in \mathrm{L}_{2}$; see Remark 2.5
} 
Riemann's work may be said to mark the beginning of a theory of the mathematically discontinuous, although there are isolated examples in Fourier's and Dirichlet's works. It planted the discontinuous firmly upon the mathematical scene. ([32, p. 116])

Thus, discontinuous functions are part of ordinary, i.e. non-set-theoretical, mathematics, predating the earliest days of set theory. Discontinuous phenomena can be modeled or represented in second-order arithmetic, but the latter cannot prove the existence of the underlying phenomena, even for the most basic case of a discontinuous function on $\mathbb{R}$. As we will see the next section, the aforementioned limitation of $L_{2}$ plays an important role in the study of splittings and disjunctions.

4.2. Discontinuity: the genesis of splittings and disjunctions. By the above, higher-order RM features lots of examples of splittings and disjunctions, esp. compared to second-order RM. We now provide a possible explanation for this observation, i.e. we answer the question why there are so many splittings and disjunctions in higher-order RM, compared to second-order RM.

First of all, it goes without saying that the language of higher-order RM is much richer than the language of second-order arithmetic. Hence, more mathematics can be expressed in $\mathrm{L}_{\omega}$, but this observation alone does not provide a satisfying explanation. The essential observation is that, as discussed in Section 4.1, second-order arithmetic cannot directly accommodate discontinuous phenomena, while higherorder arithmetic of course can. Thus, sentences of $\mathbf{L}_{\omega}$ can be divided in the following three natural categories:

(a) Sentences implying the existence of discontinuous objects.

(b) Sentences implying the continuity of a certain class of objects.

(c) Sentences that are 'neutral' regarding continuity, i.e. consistent with all sentences from items (미) and (b) .

Items (a) and (b) are often connected: while $\exists^{2}$ is discontinuous, $\neg\left(\exists^{2}\right)$ implies that all functions on $\mathbb{R}$ are continuous by [34, Prop. 3.12]. In light of the results in [30, the connection between items (a) and (b) even exists in constructive mathematics. Moreover, since it implies $\neg\left(\exists^{2}\right), \neg$ WKL belongs to item (b) , while WKL belongs to item (IC). Thus, $L_{2}$ is not restricted to (IC), but $L_{2}$ just lacks the expressiveness to state the 'logical consequences' of $\neg$ WKL, namely $\neg\left(\exists^{2}\right)$ and that all functions on $\mathbb{R}$ are continuous. To fully appreciate the previous, the reader should now consult the final parts of the proofs of Corollaries 3.10 and 3.12

Secondly, many of the results in the previous sections can be viewed as splitting sentences of $\mathrm{L}_{\omega}$ in weaker (and generally independent) components from items (a) or (b), and ( $(\mathbb{C})$. For instance, the trichotomy in the previous paragraph suggests the following way of obtaining splittings: from a sentence $A$ from item (国) (resp. item (b)), derive a weaker sentence $B$ expressing some discontinuity (resp. continuity) property, and a weaker sentence $C$ from item (C); $B$ and $C$ should be strong enough to guarantee $A \leftrightarrow[B+C]$. This kind of splitting is obtained in Sections 3.1 3.3.

Third, a different but related approach is based on the law of excluded middle, $\left(\exists^{2}\right) \vee \neg\left(\exists^{2}\right)$ in particular; other instances are used as well, but the idea is the same, as follows: starting from a relatively weak principle $C,\left(\exists^{2}\right) \vee \neg\left(\exists^{2}\right)$ implies $D \vee E$,

\footnotetext{
${ }^{8}$ While $\exists^{2}$ is the 'archetype' of a discontinuous function, Gandy's 'superjump', introduced in 23], has a characterisation in terms of discontinuities, as discussed in 25].
} 
where $D, E$ are (much) stronger than $C$. In particular, in case $\neg\left(\exists^{2}\right)$, all functions on the reals are continuous, and uncountable covers then reduce to countable ones. Hence, the Lindelöf lemma becomes trivial, while Heine-Borel compactness as in HBU simply follows from WKL, and MUC follows from FF, i.e. we obtain a (hard to prove) sentence from item (ㄷ), or a sentence from item (b). In case $\left(\exists^{2}\right)$, we are obviously in item (国). Since $D$ and $E$ both imply $C$, we obtain $C \leftrightarrow[D \vee E]$. This kind of disjunction is obtained in Sections 3.3 3.5.

In conclusion, $\mathrm{L}_{\omega}$ can represent discontinuous objects directly, in contrast to the indirect approach provided by $\mathrm{L}_{2}$. This particular richness gives rise to the trichotomy above in items (国)-(드. . Along the lines of the latter, one can obtain plenty of splittings and disjunctions in $\mathrm{L}_{\omega}$, based on the previous two paragraphs. In other words, the lack of splittings and disjunctions in classical RM is due to the weak expressive power of $\mathrm{L}_{2}$, in particular the fact that it can only model, but not prove, discontinuous phenomena.

Finally, splittings and disjunctions are not the only example of the impact of the limitations of $L_{2}$. The following remark presents another one.

Remark 4.1 (Explosions in higher-order arithmetic). We say that two sentences $A, B$ cause an 'explosion' if $A+B$ is much stronger than $A$ or $B$ considered separately (say in $\mathrm{RCA}_{0}^{\omega}$ ). We show in this remark that there are natural explosions in $\mathrm{L}_{\omega}$, which disappear in the absence of discontinuous functions.

First of all, both $\left(\exists^{2}\right)$ and HBU are weak in isolation, i.e. conservative over $\mathrm{ACA}_{0}$, but the combination implies $\mathrm{ATR}_{0}$ by [48, $\left.\S 6\right]$; see also [50, $\left.\S 3\right]$. However, $\mathrm{ACA}_{0}+\mathrm{HBU}$ is conservative 9 over $\mathrm{ACA}_{0}$.

Secondly, $\left(\exists^{2}\right)$ and the Lindelöf lemma for $\mathbb{N}^{\mathbb{N}}$, denoted $\operatorname{LIND}\left(\mathbb{N}^{\mathbb{N}}\right)$ in [51, are weak in isolation, i.e. conservative over $\mathrm{ACA}_{0}$, but the combination implies $\Pi_{1}^{1}-\mathrm{CA}_{0}$ by [51, §5]. However, $A C A_{0}+\operatorname{LIND}\left(\mathbb{N}^{\mathbb{N}}\right)$ is conservative 10 over $A_{C A}$.

The previous two explosions show that the presence of discontinuous functions has a great impact on the logical strength of (uncountable) covering theorems.

4.3. To be or not to be continuous. The results in Section 3 and 48 51] identify huge differences between second- and higher-order RM. As discussed in the previous section, these results trace back to the fact that higher-order (resp. second-order) arithmetic can (resp. cannot) directly represent discontinuous phenomena. Hence, the question arises whether one should adopt the higher-order framework instead of second-order arithmetic for the formalisation of mathematics.

In this section, we argue that one must adopt the higher-order framework in either of the following situations:

(a) if one wants to formalise mathematics in a way close to the original,

(b) the second-order formalisation should be faithful in scope to the original.

As we will see, the caveat in item (b) can be summarised as faithfulness is hard. To be absolutely clear, 'faithful' means that the second-order formalisation has the same scope or generality as the original, i.e. we are not implying that the formalisation 'should look (exactly) like the original'. The caveat in item (a) does discuss this idea of 'close to the original', and is actually inspired by the development of the gauge integral, which we discuss first, as follows.

\footnotetext{
${ }^{9}$ The ECF-interpretation translates HBU to WKL, and the latter follows from $\mathrm{ACA}_{0}$.

${ }^{10}$ The ECF-interpretation translates all versions of the Lindelöf lemma to trivialities.
} 
The gauge integral is a generalisation of the Lebesgue and (improper) Riemann integral; this integral was introduced by Denjoy ([17]), in a different and more complicated form, around the same time as the Lebesgue integral; the reformulation of Denjoy's integral by Henstock and Kurzweil in Riemann-esque terms (See 5 , p. 15]), provides a direct and elegant formalisation of the Feynman path integral ([13, 44, 46]) and financial mathematics ([45, 46]). In a nutshell, the gauge integral is just the Riemann integral with the constant ' $\delta \in \mathbb{R}^{+}$' in the usual $\varepsilon$ - $\delta$-definition replaced by a function $\delta: \mathbb{R} \rightarrow \mathbb{R}^{+}$, a small but significant change.

Now, the first step in the development of the gauge integral is always to show that this integral is well-defined, using the Cousin lemma, which implies HBU. As shown in [50, §3], $\left(\exists^{2}\right)$ and HBU are essential for the development of the gauge integral (5]) in that the former are equivalent to various basic properties of the gauge integral. Furthermore, Cousin's lemma from [15, p. 22] dates back 11] about 135 years. Thus, $\left(\exists^{2}\right)$ and HBU should count as 'core' or 'ordinary' mathematics.

The previous observations will give rise to different reactions in different people: one person will see the above as a convincing argument for the adoption of higherorder arithmetic, while another person will see this as another subject that needs to be formalised in $L_{2}$. To avoid a deadlock, we recall the connection between physics and the gauge integral from [50, §3.3] as follows: Muldowney has expressed the following opinion in a private communication.

There are a number of different approaches to the formalisation of Feynman's path integral. However, if one requires the formalisation to be close to Feynman's original formulation, then the gauge integral is really the only approach.

Arguments for this opinion, including major contributions to Rota's program for the Feyman integral, may be found in [47, §A.2]. We adopt a similar stance regarding the adoption of higher-order arithmetic: anyone interested in a direct 12 logical formalisation of the gauge integral, has no choice but to adopt the higher-order framework. In other words, assuming one wants to formalise the gauge integral in a way close to the original, one is wedded to $\left(\exists^{2}\right)$ and HBU. To be clear, this does not exclude the possibility of alternative formalisations in $L_{2}$, at the cost of a development that is (very) different from the literature. Nonetheless, the treatment in 67] is ultimately based on fundamental results of the gauge integral from [61.

We now turn to item (b), introduced at the beginning of this section and summarised as faithfulness is hard. First of all, we provide an example where it is easy (in terms of logical strength) to show that the second-order formalisation in RM is faithful in scope and generality to the original.

Example 4.2 (Coding continuous functions). As is well-known, continuous functions are represented by codes in RM (see [58, II.6.1]). It is then a natural question whether codes actually capture all continuous functions (say in a weak system). Indeed, if codes only captured a special sub-class, then a theorem of RM would be

11 The collected works of Pincherle contain a footnote by the editors (See [54, p. 67]) which states that the associated Teorema (published in 1882) corresponds to the Heine-Borel theorem. Moreover, Weierstrass proves the Heine-Borel theorem (without explicitly formulating it) in 1880 in [69] p. 204]. A detailed motivation for these claims may be found in [38 p. 96-97].

${ }^{12}$ In both second- and higher-order RM, real numbers are represented by Cauchy sequences, but the associated practice is actually close to mathematical practice, as discussed in Remark 2.4 
about that sub-class, and not about all continuous functions. However, Kohlenbach has shown in [35, §4] that WKL suffices to prove that every continuous function has a code. Hence, the RM of WKL does not really change if we introduce codes, i.e. there is a perfect match between the theorems in second- and higher-order arithmetic. Thus, second-order WKL (working in $\mathrm{RCA}_{0}^{\omega}$ ) proves that the the second-order formalisation is faithful in scope to the original.

Secondly, we provide an example where it is extremely hard to show that the second-order formalisation is faithful in scope to the original.

Example 4.3 (Coding measurable functions). Measurable functions are represented in RM by sequences of codes for continuous functions (see [58, X.1.11]). As in Example 4.2, it is a natural question whether codes actually capture all measurable functions (again in a weak system). Indeed, if codes only captured a special sub-class, then a theorem of RM would be about that sub-class, and not about all measurable functions. Now, Lusin's theorem (see e.g. 63, 1.3.28]) guarantees that every measurable function can be approximated by a sequence of continuous functions. However, as shown in [53, Lusin's theorem (and the same for many similar approximation theorems) implies WHBU from Section 3.5.2, and the latter is not provable in $\Pi_{k}^{1}-\mathrm{CA}_{0}^{\omega}$ for any $k$, i.e. $\left(\exists^{3}\right)$ is required as for $\mathrm{HBU}$ (see also [51]).

In light of Example 4.3, to guarantee that theorems about codes for measurable functions have the same generality as theorems about measurable functions, i.e. to show that the second-order formalisation is faithful to the original, we require WHBU, a third-order theorem only provable in full second-order arithmetic $Z_{2}^{\Omega}$.

Finally, while item (a) can be dismissed as an aesthetic preference, dismissing item (b) as unimportant betrays a certain formalist view of the foundations of mathematics. We finish this section with a quote on the adequacy of $L_{2}$.

We focus on the language of second order arithmetic, because that language is the weakest one that is rich enough to express and develop the bulk of core mathematics. ([58, Preface])

In conclusion, we believe Simpson's claim is wrong in the situations described by items (a) and (b) above. Indeed, mathematics is (and has been for a long time) replete with discontinuous phenomena and, in our opinion, indirectly dealing with the latter via codes is not satisfactory as this obfuscates a number of interesting mathematica 13 phenomena, like the plethora of splittings and disjunctions from Section 3 and the 'explosions' from Remark 4.1. Moreover, the requirement that e.g. codes capture all measurable functions is based on Lusin's theorem and hence WHBU, only provable in full second-order arithmetic $Z_{2}^{\Omega}$. In this light, one might as well work directly in higher-order arithmetic.

4.4. The bigger picture. We discuss the place occupied by higher-order RM in the grand scheme of things, esp. how higher-order arithmetic relates to fields based on second-order arithmetic, like RM and (classical) computability theory. We start with some historical considerations, leading up to our conclusion.

\footnotetext{
${ }^{13}$ In the basic development of the gauge integral (61] $)$, to show that the latter is well-defined, one applies the Cousin lemma (and hence HBU) to the canonical cover associated to the gauge function; the latter is continuous if and only if the original function is Riemann integrable. In other words, viewing the gauge integral as an extension of the Riemann integral, one essentially always works with uncountable covers generated by discontinuous functions. Thus, the first explosion in Remark 4.1 is quite natural from this (mathematical) point of view.
} 
If the history of (the foundations of) mathematics teaches us anything, it is that foundational topics can be quite emotionally charged. Let us therefore start with a clear caveat: there is nothing wrong with second-order arithmetic, RM and its coding, or classical computability theory. These are extremely interesting and equally successful enterprises, and perhaps therein lies the nature of the issue we wish to discuss in this section, as follows.

The aforementioned issue has a proud ancestry, and discussing an example will hopefully clarify things. The issue at hand is that successful theories (models/techniques/...) that go unchallenged for a long time develop an air of being mostly finished or complete, i.e. the grand underlying principles are know, and the rest is simple refinement. For instance, in the case of late 19th century physics, the following quote from the Nobel-prize winner Michelson is telling:

While it is never safe to affirm that the future of Physical Science has no marvels in store even more astonishing than those of the past, it seems probable that most of the grand underlying principles have been firmly established and that further advances are to be sought chiefly in the rigorous application of these principles to all the phenomena which come under our notice. (see [39-41])

Weinberg discusses this topic in [70 and sorts myth from fact, recounting quotes from Planck and Millikan that back Michelson's view. It is a matter of the historical record that only a couple of decades after Michelson's quote, modern physics was developed, yielding an entire array of new 'grand underlying principles'.

Coming back to mathematics, we believe that the history of second-order arithmetic and associated fields like RM and (classical) recursion theory has been similar: this development was extremely successful and impressive, leading to a feeling that the grand underlying principles had been firmly established. Indeed, the Gödel hierarchy is a collection of logical systems ordered via consistency strength, or essentially equivalent: ordered via inclusion 14. This hierarchy is claimed to capture most systems that are natural or have foundational import, as follows.

It is striking that a great many foundational theories are linearly ordered by $<$. Of course it is possible to construct pairs of artificial theories which are incomparable under $<$. However, this is not the case for the "natural" or non-artificial theories which are usually regarded as significant in the foundations of mathematics. ([59])

Burgess and Koellner corroborate this claim in [12, §1.5] and [33, §1.1]. The Gödel hierarchy is a central object of study in mathematical logic, as e.g. argued by Simpson in [59, p. 112] or Burgess in [12, p. 40]. Precursors to the Gödel hierarchy may be found in the work of Wang ([68]) and Bernays (see [6], and the translation in [9]). Friedman ([22]) studies the linear nature of the Gödel hierarchy in detail.

In contrast to the aforementioned 15 'received view', and starting with the results in [50,51, a large number of natural theorems (of higher-order arithmetic) have

\footnotetext{
${ }^{14}$ Simpson and Friedman claim that inclusion and consistency strength yield the same Gödel hierarchy as depicted in [59. Table 1] with the caveat that e.g. $\mathrm{RCA}_{0}$ and $\mathrm{WKL}_{0}$ have the same first-order strength, but the latter is strictly stronger than the former.

${ }^{15}$ Simpson's above grand claim notwithstanding, there are some examples of theorems (predating HBU and [50]) that also fall outside of the Gödel hierarchy (based on inclusion), like special cases of Ramsey's theorem and the axiom of determinacy from set theory ( $27[42]$ ).
} 
been identified forming a branch independent of the medium range of the Gödel hierarchy (based on inclusion 14 ). Results pertaining to 'uniform' theorems are in [51, while the results pertaining to $\mathrm{HBU}$ and the gauge integral are in [50]. We draw the following conclusions from these observations.

(1) Stepping outside $L_{2}$, as motivated in Section 4.3 yields a picture completely different from the Gödel hierarchy. This linear order is an artifact of the 'absence of discontinuity' discussed in Sections 4.1 and 4.2 ,

(2) Notions of continuity and discontinuity successful in first- and second-order arithmetic have to be rethought entirely, or abandoned for new notions, to penetrate structures in higher types, and that this remains for the future as mathematics inevitably evolves.

(3) By Example 4.3. one needs to accept hard-to-prove theorems of higherorder arithmetic to guarantee that the associated second-order formalisation is faithful. While the latter enterprise is therefore no less interesting, Simpson's claim pertaining to the adequacy of $L_{2}$ become untenable.

(4) We conjecture the existence of other branches, independent of both the Gödel hiearchy and the branch populated by HBU and its kin.

Finally, the reader should read nothing but simple analogy in the above observations: the discovery of modern physics does not compare in any way to recent discoveries in higher-order arithmetic.

\section{Conclusion}

The following table summarises some of our results, without mentioning the base theory; the latter is generally conservative over $\mathrm{WKL}_{0}$ (or is weaker). In light of this, we may conclude that the higher-order framework yields plenty of equivalences for disjunctions and splittings, in contrast to the second-order framework, and this for the reasons discussed in Section 4.2 .

\begin{tabular}{|c|c|c|}
\hline MUC $\leftrightarrow\left[\mathrm{WKL}+\left(\kappa_{0}^{3}\right)+\neg\left(\exists^{2}\right)\right]$ & $\left(\exists^{3}\right) \leftrightarrow\left[\left(Z^{3}\right)+\left(\exists^{2}\right)\right]$ & $\left(\kappa_{0}^{3}\right) \leftrightarrow\left[\left(Z^{3}\right)+\mathrm{FF}\right]$ \\
MUC $\leftrightarrow\left[\mathrm{WKL}+\left(\kappa_{0}^{3}\right)+\neg\left(S^{2}\right)\right]$ & $\left(\exists^{3}\right) \leftrightarrow\left[\left(\kappa_{0}^{3}\right)+\left(\exists^{2}\right)\right]$ & {$\left[\left(\kappa_{0}^{3}\right)+\mathrm{WKL}\right] \leftrightarrow\left[\left(\exists^{3}\right) \vee \mathrm{MUC}\right]$} \\
$\mathrm{MUC} \leftrightarrow\left[\mathrm{WKL}+\left(\kappa_{0}^{3}\right)+\neg\left(\exists^{3}\right)\right]$ & $\left(\exists^{3}\right) \leftrightarrow\left[\mathrm{FF}+\left(Z^{3}\right)+\neg \mathrm{MUC}\right]$ & $\mathrm{FF} \leftrightarrow\left[\left(\exists^{2}\right) \vee \mathrm{MUC}\right]$ \\
$\mathrm{MUC} \leftrightarrow\left[\mathrm{FF}+\neg\left(\exists^{2}\right)\right]$ & $\left(\exists^{2}\right) \leftrightarrow[\mathrm{UATR} \vee \neg \mathrm{HBU}]$ & $\mathrm{FF} \leftrightarrow\left[\left(\exists^{2}\right) \vee\left(\kappa_{0}^{3}\right)\right]$ \\
MUC $\leftrightarrow\left[\mathrm{FF}+\left(Z^{3}\right)+\neg\left(S^{2}\right)\right]$ & $\left(\exists^{2}\right) \leftrightarrow[\mathrm{FF}+\neg \mathrm{MUC}]$ & $\left(Z^{3}\right) \leftrightarrow\left[\left(\exists^{3}\right) \vee \neg\left(\exists^{2}\right)\right]$ \\
MUC $\leftrightarrow\left[\mathrm{FF}+\left(Z^{3}\right)+\neg\left(\exists^{3}\right)\right]$ & WKL $\leftrightarrow\left[\left(\exists^{2}\right) \vee \mathrm{HBU}\right]$ & $\left(Z^{3}\right) \leftrightarrow\left[\left(\exists^{3}\right) \vee \neg \mathrm{FF} \vee \mathrm{MUC}\right]$ \\
$\mathrm{T}_{1} \leftrightarrow\left[\mathrm{T}_{0} \vee \Sigma_{2^{0}}^{0} \mathrm{IND}\right]$ & WWKL $\leftrightarrow\left[\left(\exists^{2}\right) \vee \mathrm{WHBU}\right]$ & LIN $\leftrightarrow[\mathrm{HBU} \vee \neg \mathrm{WKL}]$ \\
\hline
\end{tabular}

Figure 1. Summary of our results

Finally, Simpson describes the 'mathematical naturalness' of logical systems as:

From the above it is clear that the [Big Five] five basic systems $\mathrm{RCA}_{0}, \mathrm{WKL}_{0}, \mathrm{ACA}_{0}, \mathrm{ATR} \mathrm{R}_{0}, \Pi_{1}^{1}-\mathrm{CA}_{0}$ arise naturally from investigations of the Main Question. The proof that these systems are mathematically natural is provided by Reverse Mathematics. ([58, I.12])

We leave it to the reader to decide if the aforementioned results bestow naturalness onto the theorems involved in the equivalences. We do wish to point out that some of the theorems in Figure 1 are natural, well-established, and date back more than a century already; see Section 4.3 for details. 
Acknowledgement 5.1. My research was supported by the John Templeton Foundation (grant ID 60842), the Alexander von Humboldt Foundation, and LMU Munich (via the Excellence Initiative and the Center for Advanced Studies of LMU). I express my gratitude towards these institutions. Opinions expressed in this paper do not necessarily reflect those of the John Templeton Foundation.

The research leading to this paper grew out of my joint project with Dag Normann, the papers [50,51 in particular. I thank Dag Normann for his valuable advice, especially regarding the properties of $\left(Z^{3}\right)$. I also thank Denis Hirschfeldt for his valuable suggestions regarding $\mathrm{T}_{0}$. I thank the anonymous referee for various helpful suggestions, esp. pertaining to Section 4. Finally, I thank Anil Nerode, Denis Hirschfeldt, and Steve Simpson for their help shaping Section 4.

\section{REFERENCES}

[1] Jeremy Avigad and Solomon Feferman, Gödel's functional ("Dialectica") interpretation, Handbook of proof theory, Stud. Logic Found. Math., vol. 137, 1998, pp. 337-405.

[2] Jeremy Avigad, Edward T. Dean, and Jason Rute, Algorithmic randomness, reverse mathematics, and the dominated convergence theorem, Ann. Pure Appl. Logic 163 (2012), no. 12, $1854-1864$.

[3] Robert Bartle, The elements of real analysis., John Wiley\&Sons. XV, 480 p., 1976.

[4] Robert Bartle and Donald Sherbert, Introduction to real analysis, Wiley, 2000.

[5] Robert Bartle, A modern theory of integration., Grad. Stud. Math. 32 (2001), xiv + 458.

[6] Paul Bernays, Sur le Platonisme Dans les Mathématiques, L'Enseignement Mathématique 34 (1935), 52-69.

[7] Michael Botsko, A Unified Treatment of Various Theorems in Elementary Analysis, Amer. Math. Monthly 94 (1987), no. 5, 450-452.

[8] David R. Belanger, $\mathrm{WKL}_{0}$ and induction principles in model theory., Ann. Pure Appl. Logic 166 (2015), no. 7-8, 767-799.

[9] Paul Benacerraf and Hilary Putnam, Philosophy of Mathematics: Selected Readings, 2nd ed., Cambridge University Press, 1984.

[10] L. E. J. Brouwer, Collected works. Vol. 1, North-Holland Publishing Co., Amsterdam, 1975. Philosophy and foundations of mathematics; Edited by A. Heyting.

[11] Emile Borel, Sur quelques points de la théorie des fonctions, Ann. Sci. École Norm. Sup. (3) 12 (1895), 9-55.

[12] John P. Burgess, Fixing Frege, Princeton Monographs in Philosophy, Princeton University Press, 2005.

[13] Frank E. Burk, A garden of integrals, The Dolciani Mathematical Expositions, vol. 31, Mathematical Association of America, Washington, DC, 2007.

[14] Georg Cantor, Gesammelte Abhandlungen mathematischen und philosophischen Inhalts, Springer-Verlag, Berlin-New York, 1980 (German). Reprint of the 1932 original.

[15] Pierre Cousin, Sur les fonctions de $n$ variables complexes, Acta Math. 19 (1895), no. 1, 1-61.

[16] Richard Dedekind, Stetigkeit und irrationale Zahlen, 6te unveränderte Aufl. Friedr. Vieweg \& Sohn. Braun-schweig, 1960 (German).

[17] A. Denjoy, Une extension de l'intégrale de M. Lebesgue., C. R. Acad. Sci., Paris 154 (1912), 859-862.

[18] Damir D. Dzhafarov, Reverse Mathematics Zoo. http://rmzoo.uconn.edu/

[19] Harvey Friedman, Some systems of second order arithmetic and their use, Proceedings of the International Congress of Mathematicians (Vancouver, B. C., 1974), Vol. 1, 1975, pp. 235-242.

[20] - Systems of second order arithmetic with restricted induction, I \& II (Abstracts), Journal of Symbolic Logic 41 (1976), 557-559.

[21] Harvey Friedman, Stephen G. Simpson, and Xiaokang Yu, Periodic points and subsystems of second-order arithmetic, Ann. Pure Appl. Logic 62 (1993), no. 1, 51-64.

[22] Harvey M. Friedman, Interpretations, According to Tarski, Interpretations of Set Theory in Discrete Mathematics and Informal Thinking, The Nineteenth Annual Tarski Lectures, http://u.osu.edu/friedman.8/files/2014/01/Tarski1052407-13do0b2.pdf 1 (2007), pp. 42 . 
[23] R. O. Gandy, General recursive functionals of finite type and hierarchies of functions, Ann. Fac. Sci. Univ. Clermont-Ferrand No. 35 (1967), 5-24.

[24] Russell A. Gordon, The use of tagged partitions in elementary real analysis, Amer. Math. Monthly 105 (1998), no. 2, 107-117.

[25] John P. Hartley, Effective discontinuity and a characterisation of the superjump, J. Symbolic Logic 50 (1985), no. 2, 349-358.

[26] Edwin Hewitt and Karl Stromberg, Real and abstract analysis, Springer, 1975. A modern treatment of the theory of functions of a real variable; Third printing; Graduate Texts in Mathematics, No. 25.

[27] Denis R. Hirschfeldt, Slicing the truth, Lecture Notes Series, Institute for Mathematical Sciences, National University of Singapore, vol. 28, World Scientific Publishing, 2015.

[28] Jeffry L. Hirst, Representations of reals in reverse mathematics, Bull. Pol. Acad. Sci. Math. 55 (2007), no. 4, 303-316.

[29] James Hunter, Higher-order reverse topology, ProQuest LLC, Ann Arbor, MI, 2008. Thesis (Ph.D.)-The University of Wisconsin - Madison.

[30] Hajime Ishihara, On Brouwer's continuity principle, Indag. Math. (N.S.) 29 (2018), no. 6, $1511-1524$.

[31] Jürgen Jost, Postmodern analysis, 3rd ed., Springer, 2005.

[32] Israel Kleiner, Excursions in the history of mathematics, Birkhäuser/Springer, New York, 2012.

[33] Peter Koellner, Large Cardinals and Determinacy, The Stanford Encyclopedia of Philosophy, 2014.

[34] Ulrich Kohlenbach, Higher order reverse mathematics, Reverse mathematics 2001, Lect. Notes Log., vol. 21, ASL, 2005, pp. 281-295.

[35] _ Foundational and mathematical uses of higher types, Reflections on the foundations of mathematics (Stanford, CA, 1998), Lect. Notes Log., vol. 15, ASL, 2002, pp. 92-116.

[36] Ernst Lindelöf, Sur Quelques Points De La Théorie Des Ensembles, Comptes Rendus (1903), 697-700.

[37] John Longley and Dag Normann, Higher-order Computability, Theory and Applications of Computability, Springer, 2015.

[38] Fyodor A. Medvedev, Scenes from the history of real functions, Science Networks. Historical Studies, vol. 7, Birkhäuser Verlag, Basel, 1991.

[39] Michelson A. A., XIX The Department of Physics, Introductory, Annual Register, University of Chicago (1894), p. 150.

[40] Some of the Objects and Methods of Physical Science, University of Chicago Quarterly Calendar 3 (Aug. 1894), no. 2, p. 15.

[41] Some of the Objects and Methods of Physical Science, The Electrical Engineer 21 (1896), no. 400, p. 9

[42] A. Montalbán and Richard A. Shore, The limits of determinacy in second-order arithmetic, Proc. Lond. Math. Soc. (3) 104 (2012), no. 2, 223-252.

[43] A. Montalbán, Open questions in reverse mathematics, Bull. Symb. Logic 17 (2011), 431-454.

[44] P. Muldowney, A general theory of integration in function spaces, including Wiener and Feynman integration, Pitman Research Notes in Mathematics Series, vol. 153, Longman Scientific \& Technical, Harlow; John Wiley \& Sons, Inc., New York, 1987.

[45] - The infinite dimensional Henstock integral and problems of Black-Scholes expectation, J. Appl. Anal. 8 (2002), no. 1, 1-21.

[46] Pat Muldowney, A modern theory of random variation, John Wiley \& Sons, 2012. With applications in stochastic calculus, financial mathematics, and Feynman integration.

[47] _ A modern theory of random variation, Wiley \& Sons, 2012.

[48] Dag Normann and Sam Sanders, Nonstandard Analysis, Computability Theory, and their connections, Submitted, Available from arXiv: https://arxiv.org/abs/1702.06556 (2017).

[49] _ , The strength of compactness in Computability Theory and Nonstandard Analysis, Submitted, Available from arXiv: https://arxiv.org/abs/1801.08172 (2018).

[50] - On the mathematical and foundational significance of the uncountable, Journal of Mathematical Logic, https://doi.org/10.1142/S0219061319500016 (2018).

[51] _ Uniformity in Mathematics, Submitted, arxiv: https://arxiv.org/abs/1808.09783 (2018). 
[52] Sam Sanders, Some nonstandard equivalences in Reverse Mathematics, Proceedings of CiE2018, Lecture notes in Computer Science, Springer (2018), pp. 10.

[53] Dag Normann and Sam Sanders, Representations in measure theory, In preparation (2019).

[54] Salvatore Pincherle, Sopra alcuni sviluppi in serie per funzioni analitiche (1882), Opere Scelte, I, Roma (1954), 64-91.

[55] Walter Rudin, Principles of mathematical analysis, 3rd ed., McGraw-Hill, 1976. International Series in Pure and Applied Mathematics.

[56] Nobuyuki Sakamoto and Takeshi Yamazaki, Uniform versions of some axioms of second order arithmetic, MLQ Math. Log. Q. 50 (2004), no. 6, 587-593.

[57] Stephen G. Simpson (ed.), Reverse mathematics 2001, Lecture Notes in Logic, vol. 21, ASL, La Jolla, CA, 2005.

[58] _ Subsystems of second order arithmetic, 2nd ed., Perspectives in Logic, CUP, 2009.

[59] Stephen G. Simpson, The Gödel hierarchy and reverse mathematics., Kurt Gödel. Essays for his centennial, 2010, pp. 109-127.

[60] John Stillwell, Reverse mathematics, proofs from the inside out, Princeton Univ. Press, 2018.

[61] Charles Swartz, Introduction to gauge integrals, World Scientific Publishing Co., Singapore, 2001.

[62] William W. Tait, Finitism, The Journal of Philosophy 78 (1981), 524-564.

[63] Terence Tao, An introduction to measure theory, Graduate Studies in Mathematics, vol. 126, American Mathematical Society, Providence, RI, 2011.

[64] — Analysis. I, 3rd ed., Texts and Readings in Mathematics, vol. 37, Hindustan Book Agency, 2014.

[65] B. Thomson, J. Bruckner, and A. Bruckner, Elementary real analysis, Prentice Hall, 2001.

[66] Anne Sjerp Troelstra, Metamathematical investigation of intuitionistic arithmetic and analysis, Springer Berlin, 1973. Lecture Notes in Mathematics, Vol. 344.

[67] Sean Walsh, Definability aspects of the Denjoy integral, Fund. Math. 237 (2017), no. 1, 1-29.

[68] Hao Wang, Eighty years of foundational studies, Dialectica 12 (1958), 466-497.

[69] K. Weierstraß, Ausgewählte Kapitel aus der Funktionenlehre, Teubner-Archiv zur Mathematik, vol. 9, BSB B. G. Teubner Verlagsgesellschaft, Leipzig, 1988.

[70] Steven Weinberg, Dreams of a final theory, Vintage Books, 1994. 